\title{
Correction to: Linear relation between leaf xylem water potential and transpiration in pearl millet during soil drying
}

\author{
Gaochao Cai • Mutez Ali Ahmed • \\ Michaela A. Dippold • Mohsen Zarebanadkouki • \\ Andrea Carminati
}

Published online: 24 January 2020

(C) Springer Nature Switzerland AG 2020

\section{Correction to: Plant and Soil \\ https://doi.org/10.1007/s11104-019-04408-z}

In the original version of this article, equations 4 and 9 unfortunately contained errors. These should be presented as follows:

$K_{\text {soil }}(\psi)=K_{\text {sat }}\left(\frac{\psi_{\text {soil }}}{\psi_{0}}\right)^{\tau}$

$P=\frac{E}{K_{\text {plant }}}-\psi_{\text {root_soil }}+\psi_{\text {offset }}$

Also, in the 2nd line of the last paragraph on Page 3: a period is missing between " ... high time resolution (10

The online version of the original article can be found at https://doi.org/10.1007/s11104-019-04408-z

G. Cai · M. A. Ahmed · M. Zarebanadkouki · A. Carminati University of Bayreuth, Bayreuth Center of Ecology and Environmental Research (BayCEER), Chair of Soil Physics, Universitätsstraße 30, 95447 Bayreuth, Germany s)" and "The detailed construction ...". It should read as "...high time resolution (10 s). The detailed construction..."

In the 12th line of the first paragraph on Page 4: the left half bracket " ( " is missing between "... around the cuvette" and "detailed wavelength was listed in Table S1". It should read as "... around the cuvette (detailed wavelength was listed in Table S1)."

In the last line of the figure caption on Page 9: "and" should be deleted.

In the 8th line of the first paragraph on Page 12: "e.G. aba" should be "e.g. ABA".

The original article has been corrected.

G. Cai $(\bowtie) \cdot$ M. A. Ahmed $\cdot$ M. A. Dippold

Biogeochemistry of Agroecosystems, University of Göttingen, 37077 Göttingen, Germany

e-mail: gaochao.cai@uni-bayreuth.de 